OPEN ACCESS

Edited by: Anna Rita Bilia, University of Florence, Italy

Reviewed by: Ali H. Eid,

American University of Beirut, Lebanon

Helen Skaltsa, National and Kapodistrian University

of Athens, Greece Stefan Gafner, American Botanical Council, United States

*Correspondence: Magalie El Haij magalie.elhajj@partner4health.com

Specialty section: This article was submitted to Ethnopharmacology, a section of the journal Frontiers in Pharmacology

Received: 13 March 2020 Accepted: 26 May 2020 Published: 09 June 2020

Citation:

El Haij M and Holst L (2020) Herbal Medicine Use During Pregnancy: A Review of the Literature With a Special Focus on Sub-Saharan Africa. Front. Pharmacol. 11:866. doi: 10.3389/fphar.2020.00866

\section{Herbal Medicine Use During Pregnancy: A Review of the Literature With a Special Focus on Sub-Saharan Africa}

\author{
Magalie El Hajj ${ }^{1,2 *}$ and Lone Holst ${ }^{3,4}$ \\ ${ }^{1}$ Centre for International Health, University of Bergen, Bergen, Nonway, ${ }^{2}$ Medical Affairs, Partner 4 Health, Paris, France, \\ ${ }^{3}$ Department of Global Public Health and Primary Care, University of Bergen, Bergen, Norway, ${ }^{4}$ Centre for Pharmacy, \\ University of Bergen, Bergen, Norway
}

Herbal medicine use has grown considerably worldwide among pregnant women, and is particularly widespread in sub-Saharan Africa. However, herbal medicines used across sub-Saharan Africa are associated with important research gaps and a lack of regulatory framework. This is particularly problematic, as herbal medicine use during pregnancy raises several concerns attributed to the herbal ingredient itself, conventional drug-herbal medicine interactions, and contamination or adulteration of herbal remedies. Moreover, several local herbal remedies used by sub-Saharan African pregnant women have never been botanically identified. In this review, an overview of the practice of herbal medicine, including the regulations, challenges and overall safety, is provided. Then, we discuss the prevalence of herbal medicine use during pregnancy across different sub-Saharan African countries, as well as the indications, adverse outcomes, and effectiveness of the most commonly used herbal medicines during pregnancy in that region.

Keywords: herbal medicine, pregnancy, sub-Saharan Africa, traditional medicine, ethnopharmacology

\section{INTRODUCTION}

Pregnancy is characterized by significant physiological changes resulting in various symptoms, such as nausea, vomiting, heartburn, and constipation. These ailments often cause pregnant women to resort to self-medication including the use of herbal medicines (John and Shantakumari, 2015). Consequently, the use of herbal medicines has grown considerably worldwide among pregnant women (Kennedy et al., 2013; Bayisa et al., 2014; John and Shantakumari, 2015). The prevalence of herbal medicine use during pregnancy varies significantly, depending on the geographic location, ethnicity, cultural traditions, and socioeconomic status (Illamola et al., 2020). In a multinational, cross-sectional, internet-based study conducted among 9,459 pregnant women from 23 countries in Western Europe $(n=3,201)$, Northern Europe $(n=2,820)$, Eastern Europe $(n=2,342)$, North America $(n=533)$, South America $(n=346)$, and Australia $(n=217), 28.9 \%$ of women reported herbal medicine use during pregnancy. Russia (69.0\%), Poland (49.8\%), and Australia (43.8\%) had the highest reported rates of herbal medicine users (Kennedy et al., 2013). The prevalence of herbal medicine use in pregnancy has also been reported in various other countries, including Iran (49.2\%), Egypt (27.3\%), Bangladesh (70.0\%), Iraq (53.7\%), Palestine (40.0\%), and Taiwan (33.6\%) (Chuang 
et al., 2009; Khadivzadeh and Ghabel, 2012; Al-Ramahi et al., 2013; Orief et al., 2014; Hwang et al., 2016; Ahmed et al., 2018a).

Previous research has shown that herbal medicine use during pregnancy is quite common across sub-Saharan Africa (Ahmed et al., 2018b). However, in several sub-Saharan African countries, there is a paucity of data on the use of herbal medicines among pregnant women. The following paper presents an in-depth literature review which aims to provide the best available information on herbal medicine (safety, regulations, and challenges). This review also identifies the prevalence of herbal medicine use during pregnancy across different sub-Saharan African countries, and summarizes information on the indications, adverse outcomes, and effectiveness of the most commonly used herbal medicines during pregnancy in that region. Herein, we reviewed both published and unpublished studies, drafted in English or French, reporting on herbal medicine use during pregnancy in any sub-Saharan African country, including observational studies, qualitative studies, and meta-analyses. A systematic literature search was conducted between December 2018 and February 2020, by searching the electronic databases MEDLINE and Google Scholar, and through checking reference lists for the identification of additional, relevant studies.

\section{CHALLENGES ASSOCIATED WITH THE HERBAL MEDICINE PRACTICE}

According to the World Health Organization, there are several challenges associated with the herbal medicine practice (Figure 1), which are related to regulatory status, assessment of safety and efficacy, quality control, safety monitoring, and lack of knowledge about herbal medicine within national drug regulatory authorities (World Health Organization, 2005).

The diversity of herbal remedies and their uses between different countries makes scientifically evaluating and regulating them very challenging (Wachtel-Galor and Benzie, 2011). Hence, in some countries, herbal medicines are subjected to rigorous manufacturing standards, while in others, they are regarded as food supplements for which therapeutic claims are prohibited. For example, in Europe, the Directive 2001/83/EC and the Directive 2004/24/EC divide herbal medicines into two categories: "well-established herbal medicinal products" and "traditional herbal medicinal products". For the first category, it is needed to demonstrate with sound bibliographic data that the herbal medicinal product has a well-established medicinal use with recognized efficacy and an acceptable level of safety. The herbal medicine product should have a history of safe and efficacious use for at least 10 years within the European Union for a well-established use registration. For the second category, manufacturers of herbal medicines are required to demonstrate that the safety and effectiveness of the traditional herbal medicinal product are based on long-standing medicinal use: 30 years worldwide, including at least 15 years in the European Union (Wachtel-Galor and Benzie, 2011). In the United States, by contrast, the Dietary Supplement Health and Education Act of 1994 classifies herbal products as dietary supplements, a product category that does not require pre-approval. Consequently, the manufacturer is responsible for ensuring that a herbal product is safe before it is marketed, and the Food and Drug Administration can only take regulatory action against any unsafe herbal product after it reaches the market. Therefore, in contrast to prescription and newer over-the-counter medications, herbal products in the United States are usually marketed without the benefit of clinical trials to demonstrate either efficacy or safety (Broussard et al., 2010). In sub-Saharan Africa, the development of national policies and regulations for herbal medicines is substantially more limited, resulting in the proliferation of both locally produced and imported herbal remedies of dubious quality, safety, and efficacy (World Health Organization, 2005). For instance, in Zambia, although the Medicines and Allied Substances Act of 2013 mandates the Zambia Medicines Regulations Authority to regulate and control the sales of herbal medicines, there is neither a system of registration nor a post-marketing surveillance system for herbal medicines (World Health Organization, 2005; Lusaka Times, 2018). Similarly, in Kenya, although the 2005 National Policy on Traditional Medicine and Medicinal Plants stressed the need to take inventory of all herbal medicines available in the country, these recommendations have yet to be implemented (Okumu et al., 2017).

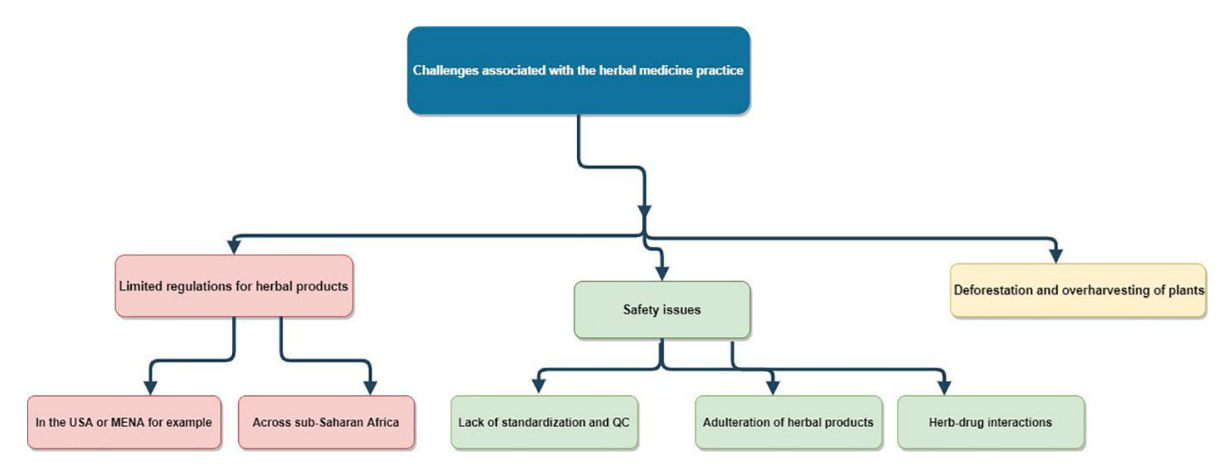

FIGURE 1 | Challenges associated with the herbal medicine practice. MENA, Middle East and North Africa; QC, quality control; USA, United States of America. 
Besides the use of inherently toxic medicinal plants, the toxicity problems of herbal medicines can be attributed to a lack of proper standardization and quality control in the formal herbal industry (Mensah et al., 2019; Ozioma and Chinwe, 2019). Indeed, there is a close correlation between the safety and efficacy of herbal medicines and the quality of the source materials used in their production. The quality of source materials is, in its turn, determined by intrinsic (genetic) and extrinsic (environmental) factors (e.g., exposure to light; availability of water; temperature of processing; period, time, and method of harvest; drying, packing, transportation, and storage of raw herbal material, etc.). Therefore, it is challenging to perform quality controls on the raw materials of herbal medicines, especially given that many countries lack operative machinery needed to implement good manufacturing practices (World Health Organization, 2005). Quality control of herbal medicines is also made more difficult by the seasonal variations in the content of the medicinal plant parts and thus the potential quantitative changes in the composition and/or ratios of bioactive ingredients (Anwar et al., 2016). In addition, there is a paucity of research on whole herbal mixtures partially because the drug approval process does not accommodate undifferentiated mixtures of natural chemicals (Wachtel-Galor and Benzie, 2011). Another source of toxicity of herbal medicines that is important to mention is adulteration of herbal products with undeclared pharmaceutical drugs and potentially toxic compounds such as harmful molecules present in other adulterating botanicals, pathogenic microorganisms, toxins, pesticides, agrochemical residues, and heavy metals (e.g., lead, mercury, and arsenic) (Saper et al., 2004; Mensah et al., 2019). Incorrect identification of plants may also lead to the inadvertent use of potentially toxic species. Furthermore, toxicity may arise as a result of herb-drug interactions (Ozioma and Chinwe, 2019). For example, it has been shown that a pharmacokinetic interaction exists between the antimalarial drug amodiaquine and Moringa oleifera Lam., a commonly used medicinal plant with multiple health benefits, when given together or following a long period of ingestion of Moringa oleifera Lam. In the presence of Moringa oleifera Lam., the peak plasma concentration of amodiaquine decreased by up to $40 \%$, as well as its area under the plasma concentration-time curve which decreased by approximately $11 \%$, leading to a significantly delayed absorption of amodiaquine (Olawoye et al., 2018). Overall, establishing herbal toxicity can be difficult. Even when herbal-related toxicity is suspected, a definitive diagnosis is difficult to establish without proper analysis of the product or plant material (Ozioma and Chinwe, 2019).

Because of the potential toxicity and safety concerns related to herbal products, there is a need to establish a pharmacovigilance system of herbal medicines that is integrated within communities and health facilities, so that data regarding their composition, preparation, indications, and adverse events can be gathered (Ahmed et al., 2018a). The latency period between the use of an herbal product and the occurrence of an adverse event should also be determined, if possible, as this can help in causality assessment in pharmacovigilance management. Such information can facilitate decisions on further protective measures to be taken concerning the future use of herbal medicines (Ozioma and Chinwe, 2019). Research is also needed to meet the challenges of identifying the active compounds in the plants, and there should be research-based evidence on the safety and efficacy of both whole herbs and extracted compounds (Wachtel-Galor and Benzie, 2011).

Regrettably, the expanding herbal product market could lead to overharvesting of plants and threaten the rich biodiversity in subSaharan Africa. In addition, poorly managed collection and cultivation practices could contribute to the extinction of endangered plant species and the destruction of natural resources (Wachtel-Galor and Benzie, 2011). It has been suggested that 15,000 medicinal plant species are threatened with extinction (Brower, 2008). Another negative consequence of this trend is that there will be essentially less choice for the future development of medicines, if the situation is not addressed (Brower, 2008). Under these circumstances, forest laws are of considerable importance, as they have a role in ensuring the sustainable availability of medicinal plants. A number of countries in sub-Saharan Africa have enacted forest laws, including the Lesotho Forestry Act of 1978, the Tanzania Forest Act of 2002, the Namibia Forest Act No. 12 of 2001, the Mozambique Forest and Wildlife Act of 1999, the South Africa National Forests Act No. 84 of 1998, the Zambia Forests Act of 1999, and the Zimbabwe Forest Amendment Act of 1999 (Moshi and Mhame, 2013). However, like other existing laws, these forest laws have deficiencies, such as failure to link the forest sector development with economic and social development objectives, weak forest administrative structures, low or poor compensation being given to the local communities that are custodians of the forest resources, poor definition of legal and institutional frameworks regarding forest management and use, and land tenure problems (Moshi and Mhame, 2013). Hence, more international efforts are needed for the preservation of plant populations.

\section{OVERALL SAFETY OF HERBAL MEDICINE DURING PREGNANCY}

The indications for the use of herbal medicines during pregnancy may vary across regions and countries, and can be mother- or child-related (Illamola et al., 2020). Herbal medicines may be used sometimes as part of maternal care to treat pregnancyrelated problems, and often to improve the well-being of the mother and/or the unborn child. The most commonly reported indications are nausea and vomiting, urinary tract infections, preparation for and/or facilitation of labor, common cold or flu, gastrointestinal problems (e.g., constipation, flatulence), pain conditions, improvement of fetal outcomes, prevention of miscarriage, relief of anxiety, treatment and/or prevention of anemia, and treatment of edema (Kennedy et al., 2013; John and Shantakumari, 2015; Ahmed et al., 2018b; Illamola et al., 2020).

As previously mentioned, safety concerns related to herbal products can be mainly attributed to the herbal ingredient itself, conventional drug-herbal medicine interactions, and 
contamination or adulteration of herbal remedies with potentially toxic compounds such as heavy metals (Broussard et al., 2010). However, herbal medicine use during pregnancy raises particular concerns, because many herbal products are specifically marketed for symptoms that occur commonly during pregnancy, such as nausea and vomiting (Allaire et al., 2000; Broussard et al., 2010). More importantly, there is a lack of randomized controlled trial data on the efficacy and safety of herbal medicine use during pregnancy. In many areas of subSaharan African, secrecy surrounds the practice of herbal medicine, with the components and preparation methods of herbal medicines, especially those intended for the treatment of serious ailments such as malaria and HIV/AIDS, exclusively residing with traditional healers (International Bioethics Committee, 2013; Ozioma and Chinwe, 2019). The fact that there are several plants used in African traditional medicine, for which little information is available on their constituents, can increase the risk of adverse reactions, particularly in vulnerable groups such as older adults, children, and pregnant women (Mensah et al., 2019; Ozioma and Chinwe, 2019). The use of unstudied herbal agents with unknown pharmacologic activity can pose a potential risk to both the pregnant women and their fetuses (Allaire et al., 2000; Ahmed et al., 2018a). In a 2004 study investigating herbal medicine use during pregnancy among 400 Norwegian women, approximately $40 \%$ of the 144 herbal medicine users consumed herbal products that were either potentially harmful or with missing information about their safety in pregnancy (Nordeng and Havnen, 2004). Similarly, in a large, multinational study which classified 126 different herbal medicines used in pregnancy according to their safety, only 28 herbs $(22.2 \%)$ were deemed as safe to use in pregnancy based on current literature (Kennedy et al., 2016). More recently, a systematic review, which aimed to determine whether herbal medicinal use during pregnancy was associated with adverse maternal or child outcomes, found that topical use of almond oil was significantly associated with preterm birth, oral raspberry leaf with caesarean delivery, and heavy liquorice consumption (greater than $500 \mathrm{mg} /$ week) with early preterm birth (Munoz
Balbontin et al., 2019). In addition, the use of herbal medicines in pregnancy constitutes a major challenge for health care providers, since pregnant women often consume herbal medicines without consulting them (Illamola et al., 2020). Hence, in order to further advance research on the benefits and adverse effects of herbal medicine use during pregnancy, it is important to determine the extent of herbal medicine use during pregnancy in different settings and to find out which herbs pregnant women use.

\section{PREVALENCE OF HERBAL MEDICINE USE DURING PREGNANCY IN SUB- SAHARAN AFRICA}

Sub-Saharan Africa is one region of the world in which herbal medicine is traditionally and culturally acknowledged (James et al., 2018b). However, evidence from sub-Saharan Africa suggests wide variability in the use of herbal medicines during pregnancy (Figure 2), from $2.0 \%$ as reported in a study conducted in the Tigray Region, Northern Ethiopia (Gebremedhin and Gomathi, 2014) to 100\% according to another study in Machakos District, Eastern Kenya (Kaingu et al., 2011). It is difficult to ascertain whether these differences in prevalence across studies are caused by variability in study design, setting, data collection, and sampling techniques or whether they represent true differences in herbal medicine use (Kennedy et al., 2013; James et al., 2018a). In a recently published systematic review by Ahmed et al. (2018b) of 50 studies, including a total of 22,404 African pregnant or lactating women, the average prevalence of herbal medicine use during pregnancy among the different African regions was between 32\% (in Central Africa) to $45 \%$ (in East Africa). This systematic review also found that herbal medicine use during pregnancy was statistically significantly associated $(p<0.05)$ with a lower educational level, increasing age, being married, low socioeconomic status, lower educational level of the spouse,

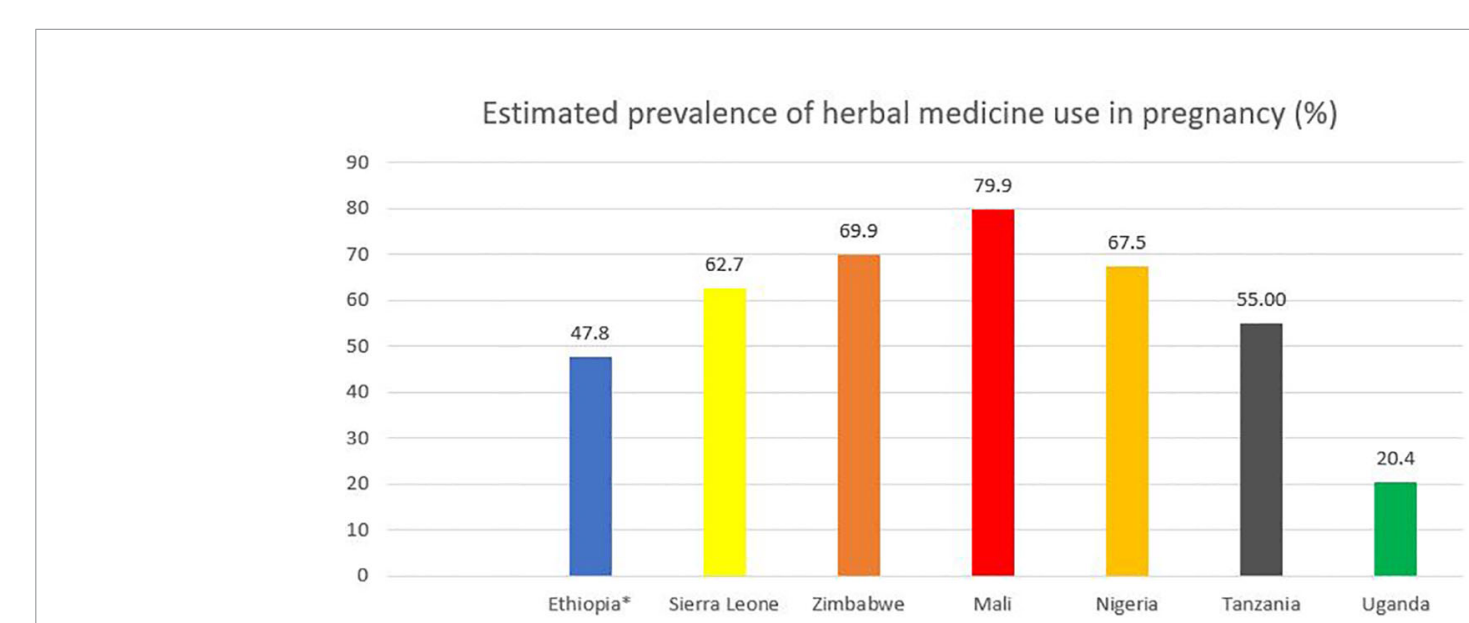

FIGURE 2 | Estimated prevalence of herbal medicine use during in pregnancy in selected sub-Saharan African countries. ${ }^{*}$ The prevalence rate for Ethiopia was based on a recent meta-analysis of eight studies (Adane et al., 2020). 
poor pregnancy outcomes, herbal medicine use in prior pregnancies, perception that medicinal plants are effective, large family size, self-employment, unemployment, and rural residence (Ahmed et al., 2018b). Table 1 illustrates the characteristics and findings of selected cross-sectional studies evaluating the use of herbal medicines among pregnant women in different sub-Saharan countries.

The popularity of herbal medicines among pregnant women can be mainly attributed to the belief that herbal products, being natural, are safe with fewer adverse events compared to conventional drugs (Ernst, 2002; Barnes et al., 2018; Peprah et al., 2019). Additionally, several studies have shown that pregnant women in sub-Saharan Africa, particularly in rural areas, use herbal medicines because they consider them less costly and more accessible than conventional drugs (Bayisa et al., 2014; Batisai, 2016; Ahmed et al., 2018a; Peprah et al., 2019). Of note, the ratio of traditional healers to the population in subSaharan Africa is 1:500, whereas the ratio of medical doctors to the population is 1:40,000, and most medical doctors are concentrated in urban areas and cities at the expense of rural areas (Abdullahi, 2011). Herbal medicine use was also shown to be in line with the sociocultural, religious and spiritual values of the people who use it in sub-Saharan Africa (Batisai, 2016; James et al., 2018b; Gakuya et al., 2020). Indeed, in many parts of subSaharan Africa, traditional medicine defines illness as a consequence of a breakdown of social balance (e.g., breaking codes of conduct in the present or in the past, ancestor or evil spirits, as well as witchcraft or fate) (Ahlberg, 2017; Stephani et al., 2018). Moreover, according to Pretorius (1991), the use of herbal medicine post-independence became one of the ways subSaharan Africans deployed to "rediscover their sociocultural identity", and to deal with inaccessible and expensive medicines foreign to them (Pretorius, 1991).

\section{MOST COMMONLY USED HERBAL MEDICINES DURING PREGNANCY IN SUB-SAHARAN AFRICA}

Due to differences in culture, traditions, and climate, it is expected that herbal medicines used during pregnancy vary across countries and regions. In the multinational study by Kennedy et al. (2013) in 9,459 pregnant women from 23 countries in Europe, North and South America, and Australia, the most frequently used herbal medicines were Zingiber officinale Roscoe (ginger), Vaccinium oxycoccus L./Vaccinium macrocarpon Aiton L. (cranberry), Valeriana officinalis L. (valerian), Rubus idaeus L. (raspberry leaf), Matricaria chamomilla L. (chamomile), and Mentha piperita L. (peppermint) (Kennedy et al., 2013). The African continent, generally known for its rich biodiversity, has an estimated total flora of over 70,000 species (Govaerts, 2001). This species richness was reflected in the systematic review by Ahmed et al. in which a total of 274 different medicinal plant species from 87 plant families were reported to be used during pregnancy
(Ahmed et al., 2018b). The most commonly cited medicinal plant species were Zingiber officinale Roscoe (ginger), Allium sativum L. (garlic), Cucurbita pepo L. (pumpkin), Gymnanthemum amygdalinum (Delile) Sch.Bip. (syn. Vernonia amygdalina Delile) (bitter leaf), Ricinus communis L. (castor oil), Garcinia kola Heckel (bitter kola), Ocimum lamiifolium Hochst. ex Benth. (basil or Dama Kesse in Amharic), Azadirachta indica A. Juss (neem), Ruta chalepensis L. (Tena Adam, in Amharic; fringed rue in English), and Aloe vera (L.) Burm.f. (Aloe vera) (Ahmed et al., 2018b). Table 2 presents the most commonly used herbal medicines during pregnancy, based on the Kennedy et al. (2013) multinational study and the Ahmed et al. (2018b) systematic review.

Several cross-sectional studies have shown that ginger is commonly used by pregnant women across sub-Saharan Africa for nausea and vomiting during pregnancy (Laelago et al., 2016; Shewamene et al., 2017; Ahmed et al., 2018b). A systematic review of 12 randomized controlled trials involving 1,278 pregnant women found that ginger significantly improved the symptoms of nausea when compared to placebo $(\mathrm{p}<0.001)$, with subgroup analyses favoring the lower daily dosage of $<1.5 \mathrm{~g}$ of ginger for nausea relief (Viljoen et al., 2014). This finding is not surprising, given that animal studies have shown that ginger can increase gastric contractility, speed up gastric emptying, and consequently increase the gastrointestinal transit time of meals, which can decrease the feeling of nausea (Chrubasik et al., 2005). However, in the systemic review by Viljoen and colleagues, ginger did not have a significant impact on vomiting episodes ( $p=0.06$ versus placebo). It was also associated with a favorable safety profile, as it did not pose a risk for spontaneous abortions or for other adverse events such as heartburn or drowsiness (Viljoen et al., 2014).

Garlic is characterized by diverse therapeutic properties, including anti-hypertensive, anti- inflammatory, anti-oxidant, anti-bacterial, hypocholesteremic, and anti-cancer properties (Al Disi et al., 2016). In a randomized, single-blind, placebocontrolled study among 100 nulliparous pregnant women, the administration of $800 \mathrm{mg} /$ day of garlic tablets during the third trimester of pregnancy was found to be effective in reducing the occurrence of hypertension ( $\mathrm{p}=0.043$ versus placebo) and in lowering serum cholesterol levels $(\mathrm{p}=0.038)$. However, compared to placebo, garlic consumption was associated with a slightly higher incidence of nausea (Ziaei et al., 2001). A metaanalysis of 18 randomized, placebo-controlled trials also found that garlic supplements induce a significant reduction in both systolic and diastolic blood pressure by 3.75 and $3.39 \mathrm{mmHg}$, respectively ( $\mathrm{p}<0.001$ compared to placebo) (Wang et al., 2015). Although the mechanism of garlic's anti-hypertensive effect remains unclear, it has been reported that garlic may elicit its anti-hypertensive effects by inhibition of angiotensin-converting enzymes through its bioactive compound allicin (Wang et al., 2015; Al Disi et al., 2016). It is recommended for pregnant women to avoid using garlic prior to surgery including caesarean section, as garlic was reported to have an anti-hemostatic effect and may consequently interfere with blood clotting (Al Disi et al., 2016; Laelago, 2018). 
TABLE 1 | Characteristics of cross-sectional studies evaluating herbal medicine use during pregnancy in sub-Saharan Africa.

\begin{tabular}{|c|c|c|c|c|c|c|c|}
\hline Reference & $\begin{array}{l}\text { Study } \\
\text { setting }\end{array}$ & $\begin{array}{l}\text { Sample } \\
\text { size }\end{array}$ & $\begin{array}{l}\text { Prevalence } \\
\text { of use }\end{array}$ & $\begin{array}{l}\text { Types of medicinal } \\
\text { plants used }\end{array}$ & Indications & Characteristics of users & $\begin{array}{c}\text { Disclosure of } \\
\text { herbal use to } \\
\text { health care } \\
\text { providers }\end{array}$ \\
\hline $\begin{array}{l}\text { (Banda } \\
\text { et al., } \\
2007)\end{array}$ & $\begin{array}{l}\text { Lusaka, } \\
\text { Zambia }\end{array}$ & 1,128 & $21 \%$ & Not reported & Not reported & $\begin{array}{l}\text { - Users were not different from non-users in } \\
\text { terms of age, education, ethnicity, or } \\
\text { income } \\
\text { - Users were more likely to drink alcohol } \\
\text { during pregnancy, have at least two sex } \\
\text { partners, engage in "dry sex", initiate sex } \\
\text { with their partner, report a previously } \\
\text { treated sexually transmitted disease, and } \\
\text { use contraception (all } p<0.01 \text { ) }\end{array}$ & $\begin{array}{l}64 \% \text { of users did } \\
\text { not want to share } \\
\text { their use of herbal } \\
\text { medicine to health } \\
\text { care providers }\end{array}$ \\
\hline $\begin{array}{l}\text { (Maluma } \\
\text { et al., } \\
\text { 2017) }\end{array}$ & $\begin{array}{l}\text { Lusaka } \\
\text { Province, } \\
\text { Zambia }\end{array}$ & 273 & $32 \%$ & $\begin{array}{l}\text { Indigenous local plants: } \\
\text { "Moono", "Makole", } \\
\text { "Mulolo", "Sope" }\end{array}$ & $\begin{array}{l}\text { Inducing or } \\
\text { accelerating labor }\end{array}$ & $\begin{array}{l}\text { - Herbal medicine use was not associated } \\
\text { with residence area, age, or education level } \\
\text { - Sociocultural beliefs were the major } \\
\text { factors that contributed to use of herbal } \\
\text { medicine during pregnancy } \\
\text { - Most users were unaware of health risks } \\
\text { associated with administering crude herbal } \\
\text { extracts during different trimesters of } \\
\text { pregnancy }\end{array}$ & Not reported \\
\hline $\begin{array}{l}\text { (James } \\
\text { et al., } \\
2018 a)\end{array}$ & $\begin{array}{l}\text { Freetown, } \\
\text { Sierra } \\
\text { Leone }\end{array}$ & 134 & $62.7 \%$ & $\begin{array}{l}\text { Luffa acutangula (L.) } \\
\text { Roxb., lime leaves (Citrus } \\
\text { aurantiifolia (Christm.) } \\
\text { Swingle), ginger }\end{array}$ & $\begin{array}{l}\text { Urinary tract } \\
\text { infections, pedal } \\
\text { edema, to improve } \\
\text { fetal outcomes }\end{array}$ & $\begin{array}{l}\text { - Pregnant women who identified as } \\
\text { Muslims were } 3.4 \text { times more likely ( } \mathrm{p}= \\
0.006) \text { than Christian women to use herbal } \\
\text { medicine } \\
\text { - Perceived effectiveness and safety of } \\
\text { herbal medicines over conventional } \\
\text { medicines }(70.2 \%) \text { was the main reason for } \\
\text { use }\end{array}$ & $\begin{array}{l}95.2 \% \text { of users did } \\
\text { not disclose their } \\
\text { herbal medicine use } \\
\text { to their conventional } \\
\text { health care } \\
\text { providers }\end{array}$ \\
\hline $\begin{array}{l}\text { (Mureyi } \\
\text { et al., } \\
\text { 2012) }\end{array}$ & $\begin{array}{l}\text { Harare, } \\
\text { Zimbabwe }\end{array}$ & 248 & $52 \%$ & $\begin{array}{l}\text { Pouzolzia mixta Sohms, } \\
\text { cocktails of unknown } \\
\text { herbs, okra (Abelmoschus } \\
\text { esculentus (L.) Moench) }\end{array}$ & $\begin{array}{l}\text { For widening of } \\
\text { birth canal, labor } \\
\text { induction, } \\
\text { nutritional } \\
\text { supplement }\end{array}$ & $\begin{array}{l}\text { - Herbal medicine use in pregnancy was } \\
\text { significantly associated with being in the } \\
20-25 \text { age group }(p=0.021) \text {, nulliparity ( } p \\
=0.004) \text {, nulligravidity }(p=0.002) \text {, and } \\
\text { residing in a high-density neighborhood ( } p= \\
0.04) \\
\text { - Almost all herbal medicine interventions } \\
\text { were employed beginning at onset of the } \\
\text { third trimester }\end{array}$ & Not reported \\
\hline $\begin{array}{l}\text { (Godlove, } \\
\text { 2011) }\end{array}$ & $\begin{array}{l}\text { Mbeya, } \\
\text { southwest } \\
\text { Tanzania }\end{array}$ & 400 & $55 \%$ & Not reported & $\begin{array}{l}\text { Labor induction, } \\
\text { to improve fetal } \\
\text { outcomes }\end{array}$ & $\begin{array}{l}\text { - The use of herbal medicines during } \\
\text { pregnancy was associated with long } \\
\text { distance to the nearest public health facility, } \\
\text { and low education level (all } p \leq 0.01 \text { ) } \\
\text { - The insufficient effectiveness of } \\
\text { conventional medicines ( } 64.1 \% \text { ) and the } \\
\text { accessibility of herbal medicines ( } 30.5 \%) \\
\text { were reported as the main reasons for use }\end{array}$ & Not reported \\
\hline $\begin{array}{l}\text { (Bayisa } \\
\text { et al., } \\
\text { 2014) }\end{array}$ & $\begin{array}{l}\text { Nekemte, } \\
\text { Western } \\
\text { Ethiopia }\end{array}$ & 250 & $50.4 \%$ & $\begin{array}{l}\text { Ginger, garlic, Tena Adam } \\
\text { (Ruta chalepensis L.), } \\
\text { eucalyptus (Eucalyptus } \\
\text { globulus Labill.) }\end{array}$ & $\begin{array}{l}\text { For treatment of } \\
\text { nausea, morning } \\
\text { sickness, } \\
\text { vomiting, cough }\end{array}$ & $\begin{array}{l}\text { - Age, educational status, marriage, } \\
\text { ethnicity, and source of information were } \\
\text { not associated with herbal medicine use } \\
\text { - About } 70 \% \text { of users were pregnant } \\
\text { women on their first trimester }\end{array}$ & Not reported \\
\hline $\begin{array}{l}\text { (Laelago } \\
\text { et al., } \\
\text { 2016) }\end{array}$ & $\begin{array}{l}\text { Hossana, } \\
\text { Southern } \\
\text { Ethiopia }\end{array}$ & 363 & $73.1 \%$ & $\begin{array}{l}\text { Garlic, ginger, Tena Adam } \\
\text { (Ruta chalepensis L.), } \\
\text { Dama Kesse (Ocimum } \\
\text { lamiifolium Hochst. ex } \\
\text { Benth.), eucalyptus } \\
\text { (Eucalyptus globulus } \\
\text { Labill.) }\end{array}$ & $\begin{array}{l}\text { Management of } \\
\text { nausea, vomiting, } \\
\text { abdominal pain, } \\
\text { common cold }\end{array}$ & $\begin{array}{l}\text { Being in the first trimester of pregnancy, } \\
\text { having less education, and having less } \\
\text { knowledge about herbal medicine favored } \\
\text { the use of medicinal plants }\end{array}$ & Not reported \\
\hline
\end{tabular}


TABLE 1 | Continued

\begin{tabular}{|c|c|c|c|c|c|c|c|}
\hline Reference & $\begin{array}{l}\text { Study } \\
\text { setting }\end{array}$ & $\begin{array}{l}\text { Sample } \\
\text { size }\end{array}$ & $\begin{array}{l}\text { Prevalence } \\
\text { of use }\end{array}$ & $\begin{array}{l}\text { Types of medicinal } \\
\text { plants used }\end{array}$ & Indications & Characteristics of users & $\begin{array}{l}\text { Disclosure of } \\
\text { herbal use to } \\
\text { health care } \\
\text { providers }\end{array}$ \\
\hline $\begin{array}{l}\text { (Mekuria } \\
\text { et al., } \\
\text { 2017) }\end{array}$ & $\begin{array}{l}\text { Gondar, } \\
\text { Northern } \\
\text { Ethiopia }\end{array}$ & 364 & $48.6 \%$ & $\begin{array}{l}\text { Ginger, Dama Kesse } \\
\text { (Ocimum lamiifolium } \\
\text { Hochst. ex Benth.) }\end{array}$ & $\begin{array}{l}\text { Common cold, } \\
\text { inflammation }\end{array}$ & $\begin{array}{l}\text { - Rural residency, having no formal } \\
\text { education, and having an average monthly } \\
\text { income }<100 \text { United States Dollars were } \\
\text { found to be strong predictors of herbal } \\
\text { medicine use } \\
\text { - } 68.4 \% \text { of users consumed herbal } \\
\text { medicines during their third trimester }\end{array}$ & $\begin{array}{l}89.8 \% \text { of users had } \\
\text { not consulted their } \\
\text { doctors about their } \\
\text { herbal medicine use }\end{array}$ \\
\hline $\begin{array}{l}\text { (Fakeye } \\
\text { et al., } \\
\text { 2009) }\end{array}$ & $\begin{array}{l}\text { North } \\
\text { Central, } \\
\text { North } \\
\text { West and } \\
\text { South } \\
\text { West, } \\
\text { Nigeria }\end{array}$ & 595 & $67.5 \%$ & Not reported & Not reported & $\begin{array}{l}\text { - Age ( } p=0.003) \text {, geographical zones }(p= \\
0.02) \text {, and educational status }(p=0.04) \\
\text { were significantly associated with herbal } \\
\text { medicine use } \\
\text { - Users used medicinal plants because they } \\
\text { perceived them as being more effective } \\
\text { than conventional medicines ( } 22.4 \%) \text {, and } \\
\text { safe }(21.1 \%) \\
\text { - } 56.6 \% \text { of participants did not support } \\
\text { combining herbal medicines with } \\
\text { conventional medications to forestall drug- } \\
\text { herb interaction }\end{array}$ & Not reported \\
\hline $\begin{array}{l}\text { (Tamuno } \\
\text { et al., } \\
\text { 2010) }\end{array}$ & $\begin{array}{l}\text { Kano, } \\
\text { North } \\
\text { West } \\
\text { Nigeria }\end{array}$ & 500 & $31.4 \%$ & Ginger, garlic & Not reported & $\begin{array}{l}\text { - Use of herbal medicine was significantly } \\
\text { associated with no formal education and } \\
\text { low socioeconomic status }(p<0.05 \text { for } \\
\text { both) } \\
\text { - Over } 40 \% \text { of women reported combined } \\
\text { use of herbs and drugs }\end{array}$ & Not reported \\
\hline $\begin{array}{l}\text { (Duru } \\
\text { et al., } \\
\text { 2016) }\end{array}$ & $\begin{array}{l}\text { Owerri, } \\
\text { South East } \\
\text { Nigeria }\end{array}$ & 500 & $36.8 \%$ & $\begin{array}{l}\text { Bitter leaf } \\
\text { (Gymnanthemum } \\
\text { amygdalinum (Delile) } \\
\text { Sch.Bip.), palm kernel oil, } \\
\text { bitter kola (Garcinia kola } \\
\text { Heckel) }\end{array}$ & Not reported & $\begin{array}{l}\text { Being married }(p<0.001) \text {, having no formal } \\
\text { education ( } p<0.001) \text {, and having a } \\
\text { monthly income }>250 \text { USD ( } p=0.003 \text { ) } \\
\text { were significantly associated with herbal } \\
\text { medicine use during pregnancy }\end{array}$ & Not reported \\
\hline $\begin{array}{l}\text { (Nergard } \\
\text { et al., } \\
\text { 2015) }\end{array}$ & $\begin{array}{l}\text { One urban } \\
\text { and two } \\
\text { rural } \\
\text { regions, } \\
\text { Mali }\end{array}$ & 209 & $79.9 \%$ & $\begin{array}{l}\text { Lippia chevalieri Moldenke, } \\
\text { Combretum micranthum } \\
\text { G. Don, Parkia biglobosa } \\
\text { (Jacq.) R.Br. ex G.Don, } \\
\text { Vepris heterophylla (Engl.) } \\
\text { Letouzey }\end{array}$ & $\begin{array}{l}\text { For general well- } \\
\text { being, to treat } \\
\text { malaria } \\
\text { symptoms, } \\
\text { edema, urinary } \\
\text { tract infection, } \\
\text { tiredness }\end{array}$ & $\begin{array}{l}\text { - Sociodemographic characteristics were } \\
\text { not associated with the use of herbal } \\
\text { medicines } \\
\text { - Frequent use of herbal medicines was } \\
\text { reported during the first semester }\end{array}$ & $\begin{array}{l}\text { Pregnant women } \\
\text { used herbal } \\
\text { preparations } \\
\text { without any } \\
\text { supervision from } \\
\text { their health care } \\
\text { providers }\end{array}$ \\
\hline $\begin{array}{l}\text { (Mothupi, } \\
\text { 2014) }\end{array}$ & $\begin{array}{l}\text { Nairobi, } \\
\text { Kenya }\end{array}$ & 333 & $12 \%$ & Not reported & $\begin{array}{l}\text { To treat } \\
\text { toothache, back } \\
\text { pain, flu, } \\
\text { indigestion, } \\
\text { swollen feet }\end{array}$ & $\begin{array}{l}\text { - The use of herbal medicine was } \\
\text { associated with a lower level of education } \\
\text { ( } p=0.007) \text {, and use before the index } \\
\text { pregnancy ( } p<0.001 \text { ) } \\
\text { - } 51 \% \text { of users reported use of combined } \\
\text { herbs with pharmaceutical drugs }\end{array}$ & $\begin{array}{l}\text { Only } 12.5 \% \text { of } \\
\text { users disclosed the } \\
\text { use of herbal } \\
\text { medicines to health } \\
\text { care professionals }\end{array}$ \\
\hline $\begin{array}{l}\text { (Nyeko } \\
\text { et al., } \\
\text { 2016) }\end{array}$ & $\begin{array}{l}\text { Gulu } \\
\text { District, } \\
\text { Northern } \\
\text { Uganda }\end{array}$ & 383 & $20.4 \%$ & Local herbs (not reported) & $\begin{array}{l}\text { To treat } \\
\text { abdominal/waist } \\
\text { pain, fever, skin } \\
\text { problems, nausea } \\
\text { and vomiting, and } \\
\text { for induction of } \\
\text { labor }\end{array}$ & $\begin{array}{l}\text { - Women who used herbal medicines in } \\
\text { their previous pregnancies were } 8 \text { times } \\
\text { more likely to use them during the current } \\
\text { pregnancy } \\
\text { - Residing more than } 5 \mathrm{~km} \text { from the nearest } \\
\text { health facility was associated with increased } \\
\text { herbal medicine }\end{array}$ & $\begin{array}{l}89.7 \% \text { of the users } \\
\text { of herbal medicines } \\
\text { did not disclose the } \\
\text { use of local herbs } \\
\text { to their health care } \\
\text { providers }\end{array}$ \\
\hline $\begin{array}{l}\text { (Adusi- } \\
\text { Poku et al., } \\
\text { 2016) }\end{array}$ & $\begin{array}{l}\text { Offinso } \\
\text { North } \\
\text { District, } \\
\text { Ghana }\end{array}$ & 384 & $6.5 \%$ & $\begin{array}{l}\text { Senna occidentalis (L.) } \\
\text { Link, Sida acuta Burm.f., } \\
\text { Cola gigantea A.Chev. }\end{array}$ & $\begin{array}{l}\text { To ease labor and } \\
\text { to improve fetal } \\
\text { outcomes }\end{array}$ & $\begin{array}{l}\text { High usage was found among married } \\
\text { women, and among those with no formal } \\
\text { education, and women with median age of } \\
25 \text { years }\end{array}$ & Not reported \\
\hline
\end{tabular}

Similarly to garlic, pumpkin is also known for its multiple beneficial effects, such as anti-diabetic, anti-hypertensive, antitumor, anti-bacterial, anti-viral, anti-fungal, hypocholesteremic, anti-ulcer, anti-inflammatory, and analgesic effects (Jafarian et al., 2012; Adnan et al., 2017). Protein-bound polysaccharides and pectines, found in different species of Cucurbita, could exert anti-oxidant effects by elevating glutathione peroxidase and superoxide dismutase activity as well as reducing serum levels 
TABLE 2 | Most commonly used herbal medicines during pregnancy: indications, reported adverse events, and preparations.

\begin{tabular}{|c|c|c|c|c|c|}
\hline $\begin{array}{l}\text { Common } \\
\text { name(s) }\end{array}$ & $\begin{array}{l}\text { Binomial name } \\
\text { (s) }\end{array}$ & Indication(s) & Reported adverse events & Common preparation(s) & References \\
\hline Ginger & $\begin{array}{l}\text { Zingiber officinale } \\
\text { Roscoe }\end{array}$ & Nausea, vomiting & $\begin{array}{l}\text { Drowsiness, reflux, vomiting, heartburn, } \\
\text { headache, abdominal discomfort, } \\
\text { preterm delivery, smaller head } \\
\text { circumference of newborns }\end{array}$ & $\begin{array}{l}\text { Ginger tea; chewing raw } \\
\text { ginger }\end{array}$ & $\begin{array}{l}\text { (Holst et al., 2011; Dante } \\
\text { et al., 2014; Trabace } \\
\text { et al., 2015) }\end{array}$ \\
\hline Cranberry & $\begin{array}{l}\text { - Vaccinium } \\
\text { oxycoccos L. } \\
\text { - Vaccinium } \\
\text { macrocarpon } \\
\text { Aiton }\end{array}$ & Urinary tract infections & $\begin{array}{l}\text { Gastrointestinal upset, spotting in the } \\
\text { second and third trimesters }\end{array}$ & Cranberry juice & $\begin{array}{l}\text { (Holst et al., 2011; } \\
\text { Heitmann et al., 2013; } \\
\text { Dante et al., 2014) }\end{array}$ \\
\hline Valerian & $\begin{array}{l}\text { Valeriana } \\
\text { officinalis L. }\end{array}$ & Sleep disorders & Diarrhea & Root decoction; capsules & (Bent et al., 2006) \\
\hline $\begin{array}{l}\text { Raspberry } \\
\text { leaf }\end{array}$ & Rubus idaeus L. & $\begin{array}{l}\text { Nausea, increase in milk } \\
\text { production, labor induction }\end{array}$ & $\begin{array}{l}\text { Hypoglycemia, higher percentage of } \\
\text { cesarean deliveries versus non-users }\end{array}$ & Raspberry leaf tea; capsules & $\begin{array}{l}\text { (Nordeng et al., 2011; } \\
\text { Al-Ramahi et al., 2013; } \\
\text { Cheang et al., 2016) }\end{array}$ \\
\hline Chamomile & $\begin{array}{l}\text { - Matricaria } \\
\text { chamomilla L. } \\
\text { - Chamaemelum } \\
\text { nobile (L.) All. }\end{array}$ & $\begin{array}{l}\text { Gastrointestinal irritation, } \\
\text { insomnia, joint pain, relaxation }\end{array}$ & $\begin{array}{l}\text { Breast engorgement and tenderness, } \\
\text { low birth weight, preterm delivery }\end{array}$ & $\begin{array}{l}\text { Chamomile tea prepared from } \\
\text { dried flowers }\end{array}$ & $\begin{array}{l}\text { (Al-Ramahi et al., 2013; } \\
\text { Trabace et al., 2015; } \\
\text { Silva et al., 2018) }\end{array}$ \\
\hline Peppermint & $\begin{array}{l}\text { Mentha piperita } \\
\text { L. }\end{array}$ & $\begin{array}{l}\text { Nausea, vomiting, flatulence, } \\
\text { indigestion, irritable bowel } \\
\text { syndrome }\end{array}$ & $\begin{array}{l}\text { Heartburn, dry mouth, belching, rash, } \\
\text { dizziness, headache }\end{array}$ & Leaf tea; oil extract & $\begin{array}{l}\text { (Al-Ramahi et al., 2013; } \\
\text { Alammar et al., 2019) }\end{array}$ \\
\hline Garlic & Allium sativum L. & $\begin{array}{l}\text { Prophylaxis of preeclampsia, } \\
\text { preterm birth prophylaxis, } \\
\text { enhancing the immune system }\end{array}$ & Foul odor, nausea & $\begin{array}{l}\text { Eating raw garlic; garlic tea; } \\
\text { garlic juice }\end{array}$ & $\begin{array}{l}\text { (Ziaei et al., 2001; Myhre } \\
\text { et al., 2013; Laelago, } \\
\text { 2018) }\end{array}$ \\
\hline Pumpkin & $\begin{array}{l}\text { Cucurbita pepo } \\
\text { L. }\end{array}$ & $\begin{array}{l}\text { Nutritional supplement, cough, } \\
\text { fever, common cold, headache, } \\
\text { heartburn, gastrointestinal } \\
\text { irritation, edema }\end{array}$ & $\begin{array}{l}\text { No adverse events were identified in } \\
\text { the literature }\end{array}$ & $\begin{array}{l}\text { Decoction of seeds; leaf juice } \\
\text { (mixed with milk) }\end{array}$ & $\begin{array}{l}\text { (Adnan et al., 2017; } \\
\text { Ahmed et al., 2018b) }\end{array}$ \\
\hline Bitter leaf & $\begin{array}{l}\text { Gymnanthemum } \\
\text { amygdalinum } \\
\text { (Delile) Sch.Bip. }\end{array}$ & $\begin{array}{l}\text { Nausea, vomiting, fever, } \\
\text { constipation, increasing appetite, } \\
\text { strengthening the pelvic floor } \\
\text { muscles, malaria, anemia }\end{array}$ & Stimulation of uterine motility & $\begin{array}{l}\text { Leaf soup; fresh leaves are } \\
\text { washed and squeezed in clean } \\
\text { water and the water is } \\
\text { extracted for drinking }\end{array}$ & $\begin{array}{l}\text { (Attah et al., 2012; } \\
\text { Olowokere and Olajide, } \\
\text { 2013; Nalumansi et al., } \\
\text { 2017) }\end{array}$ \\
\hline $\begin{array}{l}\text { - Castor oil } \\
\text { - Castor } \\
\text { bean }\end{array}$ & $\begin{array}{l}\text { Ricinus } \\
\text { communis L. }\end{array}$ & Inducing labor & $\begin{array}{l}\text { Nausea, abdominal pain, uterine } \\
\text { rupture }\end{array}$ & $\begin{array}{l}\text { Leaves or roots soaked in hot } \\
\text { or cold water, usually drank at } \\
\text { labor onset }\end{array}$ & $\begin{array}{l}\text { (Sicuranza and Figueroa, } \\
\text { 2003; Dante et al., 2014; } \\
\text { Gilad et al., 2018) }\end{array}$ \\
\hline Bitter kola & $\begin{array}{l}\text { Garcinia kola } \\
\text { Heckel }\end{array}$ & Nausea, vomiting & $\begin{array}{l}\text { Weight loss, prolonged sleep duration, } \\
\text { increased libido }\end{array}$ & Chewing the seeds & $\begin{array}{l}\text { (Adegbehingbe et al., } \\
\text { 2008; Ahmed et al., } \\
\text { 2018b) }\end{array}$ \\
\hline $\begin{array}{l}\text { - Dama } \\
\text { Kesse (in } \\
\text { Amharic) } \\
\text { - Basil }\end{array}$ & $\begin{array}{l}\text { Ocimum } \\
\text { lamiifolium } \\
\text { Hochst. ex } \\
\text { Benth. }\end{array}$ & $\begin{array}{l}\text { Headache, fever, inflammation, } \\
\text { joint pain, back pain, common } \\
\text { cold, cough, eye infections }\end{array}$ & $\begin{array}{l}\text { No adverse events were identified in } \\
\text { the literature }\end{array}$ & $\begin{array}{l}\text { Oral decoction of crushed } \\
\text { leaves; leaf juice drank or } \\
\text { sniffed or used as an eye drop }\end{array}$ & $\begin{array}{l}\text { (Hailemeskel et al., } \\
\text { 2017; Nega et al., 2019) }\end{array}$ \\
\hline $\begin{array}{l}\text { - Neem } \\
\text { - Nimtree } \\
\text { - Indian } \\
\text { lilac }\end{array}$ & $\begin{array}{l}\text { Azadirachta } \\
\text { indica A. Juss }\end{array}$ & $\begin{array}{l}\text { Inducing labor, malaria, pain, } \\
\text { hemorrhoids, enhancing fetal } \\
\text { development }\end{array}$ & $\begin{array}{l}\text { Vomiting, metabolic acidosis, } \\
\text { encephalopathy }\end{array}$ & $\begin{array}{l}\text { Body smeared with mashed } \\
\text { leaves; neem leaf tea; oral } \\
\text { neem bark extracts }\end{array}$ & $\begin{array}{l}\text { (Mishra and Dave, 2013; } \\
\text { Malan et al., 2015; Dika } \\
\text { et al., 2017; Ahmed } \\
\text { et al., 2018b) }\end{array}$ \\
\hline $\begin{array}{l}\text { - Tena } \\
\text { Adam (in } \\
\text { Amharic) } \\
\text { - Fringed } \\
\text { rue }\end{array}$ & $\begin{array}{l}\text { Ruta chalepensis } \\
\text { L. }\end{array}$ & $\begin{array}{l}\text { Nausea, vomiting, common cold, } \\
\text { abdominal discomfort }\end{array}$ & Sedation, drowsiness & Leaf tea; leaf juice & $\begin{array}{l}\text { (Al-Said et al., 1990; } \\
\text { Ahmed et al., 2018b) }\end{array}$ \\
\hline Aloe vera & $\begin{array}{l}\text { Aloe vera (L.) } \\
\text { Burm.f. }\end{array}$ & $\begin{array}{l}\text { Digestive problems, constipation, } \\
\text { skin treatment }\end{array}$ & Itching, rash & $\begin{array}{l}\text { Topical gel; lotion; leaf tea; leaf } \\
\text { juice }\end{array}$ & $\begin{array}{l}\text { (Cuzzolin et al., 2010; } \\
\text { Facchinetti et al., 2012; } \\
\text { Ahmed et al., 2018b) }\end{array}$ \\
\hline
\end{tabular}

of malondialdehyde (Mahmoodpoor et al., 2018). In addition, enhancement of splenic lymphocyte proliferation, natural killer cell activity, and an increase in the number of $\mathrm{CD}^{+}, \mathrm{CD}^{+}{ }^{+} \mathrm{T}$ cells, and the $\mathrm{CD}^{+} / \mathrm{CD}^{+}$ratio by pumpkin extracts have been reported, confirming the immuno-modulatory activity of pumpkin (Xia et al., 2003). Despite the long-standing medicinal use of pumpkin, there are no clinical trial data available to support its use during pregnancy and lactation.

Bitter leaf is a perennial plant growing predominantly in tropical Africa. It has gained wide application in Nigeria, Uganda, Ethiopia, Tanzania, and other sub-Saharan African countries for the treatment of malaria, amoebiasis, measles, 
and helminthic infections, and its use is quite popular among pregnant women (Olowokere and Olajide, 2013; Nalumansi et al., 2017; Oyeyemi et al., 2018). In a single-arm trial from Western Uganda examining the efficacy and safety of an oral bitter leaf infusion in patients aged 12 years and over with uncomplicated malaria, bitter leaf was associated with an adequate clinical response after 2 weeks of treatment in $67 \%$ of cases, as well as reduced parasitemia by $32 \%$. No significant adverse events were reported throughout the trial (Challand and Willcox, 2009). Although more than 30 bioactive compounds have been isolated, the sesquiterpene lactones found in the leaves of Gymnanthemum amygdalinum have been shown to be the main active compounds responsible for most of the plant's therapeutic activities (Luo et al., 2011; Abay et al., 2015; Oyeyemi et al., 2018). Despite the absence of experimental studies in pregnant women, a 3-week experiment among 20 pregnant rats found that the aqueous extract of bitter leaf has an abortifacient effect, and is associated with reduced serum progesterone levels. The same study also found a median lethal dose above $5,000 \mathrm{mg} / \mathrm{kg}$, prompting the authors to conclude that bitter leaf is safe for human consumption (Isa et al., 2014). Nevertheless, caution is advised with the clinical use of bitter leaf during pregnancy, and particularly during the first trimester, as it may stimulate uterine contractions (Olowokere and Olajide, 2013).

There is a wide use of castor oil to induce labor. In a recent systematic review and meta-analysis of five randomized controlled trials and five observational studies aimed at comparing pregnancy outcomes between users and non-users of castor oil for labor induction, castor oil users were significantly more likely to give birth within $24 \mathrm{~h}$ than non-users (relative risk, 3.46; 95\% confidence interval, 1.58-7.55), highlighting the oxytocic properties of this medicinal plant (Zamawe et al., 2018). Castor oil is thought to induce labor via its main ingredient ricinoleic acid, a hydroxylated fatty acid released from castor oil by intestinal lipases. Ricinoleic acid specifically activates the prostaglandin $\mathrm{EP}_{3}$ receptor resulting in laxation and uterus contraction (Tunaru et al., 2012). With respect to safety, the systematic review by Zamawe and colleagues found no statistically significant differences in the rate of hemorrhage, caesarean section, assisted vaginal delivery, referral to neonatal intensive care unit, meconium-stained liquor, maternal death, stillborn, and uterine rupture between users and non-users of castor oil (Zamawe et al., 2018). Nevertheless, caution should be exercised, as cases of uterine rupture associated with castor oil ingestion have been reported in the literature (Sicuranza and Figueroa, 2003; Fruscalzo et al., 2012).

Bitter kola is a flowering plant found mostly in the tropical rain forest region of Central and West Africa. Although bitter kola is mostly used among pregnant women to treat nausea and vomiting (Ahmed et al., 2018b; Laelago, 2018), reported pharmacological effects of this plant include anti-diabetic, antiinflammatory, antipyretic, immunomodulatory, antiatherogenic, anti-microbial, and hepaprotective effects (Okoye et al., 2014). To our knowledge, there are no available clinical trial data on bitter kola in pregnant women. However, a randomized, placebo-controlled, double-blind study of bitter kola in patients with knee osteoarthritis reported a favorable safety profile of bitter kola, with only minor adverse events (i.e., weight loss, prolonged sleep duration, and increased libido) (Adegbehingbe et al., 2008). These adverse events are not surprising, given that bitter kola seeds contain approximately $3 \%$ of caffeine and $2 \%$ of theobromine. Given their relatively high caffeine content, pregnant women should limit their use.

Dama Kesse is a versatile aromatic genus that is particularly popular in Ethiopia, with anti-malarial, antipyretic, anti-oxidant, anti-spasmodic, and anti-bacterial properties (Kefe et al., 2016; Ahmed et al., 2018b; Sahalie et al., 2018). Despite its widespread use among pregnant women across Ethiopia (Adane et al., 2020), Dama Kesse is very poorly studied, with no clinical studies identified.

Neem is a fast-growing tree that is a member of the Meliaceae family. It is characterized by a broad spectrum of activity including anti-inflammatory, antipyretic, analgesic, cardiovascular, hypoglycemic, diuretic, immunomodulatory, dermatological, anti-bacterial, anti-fungal, anti-ulcer, antitumor, and anti-malarial effects. Although its exact mechanism in the prevention of pathogenesis is not entirely understood, the therapeutic activity of neem is thought to result from the synergistic/additive effects of its various bioactive compounds such as azadirachtin, nimbolinin, nimbin, nimbidin, nimbidol, sodium nimbinate, gedunin, salannin, and quercetin (Govindachari et al., 1998; Alzohairy, 2016). Despite being a native to the Indian subcontinent, neem is commonly used across sub-Saharan Africa, particularly for malaria prevention and treatment (Luong et al., 2012; Kumar and Navaratnam, 2013; Malan et al., 2015). Although neem has been extensively investigated in the non-clinical setting, only a handful of clinical studies have been published, of which none were conducted in pregnant women (Alzohairy, 2016). In a small, single-arm clinical trial among 26 adult patients suffering from gastric acidity and gastroduodenal ulcers, the twice daily administration of 30-mg capsules of neem bark extract for 10 days was associated with a significant decrease of gastric acid secretion (by 77\% compared to baseline; $\mathrm{p}<0.002$ ). The volume of gastric secretion and pepsin activity were also inhibited by $63 \%$ and $50 \%$, respectively. Neem showed a favorable safety profile, with no major adverse events reported (Bandyopadhyay et al., 2004). In another small, 6-week trial among 36 patients with oral infections, neem extract dental gel significantly reduced the plaque index and bacterial count compared to those receiving chlorhexidine gluconate $(0.2 \% \mathrm{w} / \mathrm{v})$ mouthwash (Pai et al., 2004). Despite these positive results, case reports in both adults and children have reported neem oil poisoning incidents causing vomiting, seizures, hepatic toxicity, metabolic acidosis, and toxic encephalopathy (Sinniah and Baskaran, 1981; Sundaravalli et al., 1982; Mishra and Dave, 2013). In addition, oral use of purified neem seed extract has been found to be efficacious in pregnancy termination in both rodents and primates, as indicated by a total decline of chorionic gonadotropin and progesterone and by resumption of cyclic changes in hormone profiles and perineal sex swelling in baboons (Talwar et al., 1997). This abortive effect 
of oral neem seed extracts seems to be propelled by activation of cell-mediated immune reactions (Atawodi and Atawodi, 2009). Hence, despite the absence of reliable clinical trial data, the use of neem preparations during pregnancy should be discouraged.

Tena Adam is a shrubby plant that is cultivated in the highlands of Ethiopia. Similarly to Dama Kesse, it is poorly studied with no clinical trial data available. However, the administration of an aqueous infusion of Tena Adam during organogenic period in pregnant mice led to increased flow and decreased vascular resistance in the placenta bed, as well as fetal thymic involution (Zeichen de Sa et al., 2000). Thus, Tena Adam should also be discouraged in pregnant women until welldesigned clinical trials are available.

Aloe vera is perhaps one of the most widely used herbal remedies for topical skin conditions. Aloe vera extracts improve skin moisture by a humectant mechanism, and prevent skin ulcers as they contain mucopolysaccharides, amino acids, zinc, and water (Dal'Belo et al., 2006; Hekmatpou et al., 2019). Although the topical application of Aloe vera is associated with infrequent adverse events (Hekmatpou et al., 2019), orally ingested Aloe vera whole leaf extract has shown clear evidence of carcinogenic activity in rats, and was classified by the International Agency for Research on Cancer as a possible human carcinogen (Guo and Mei, 2016). In addition, pregnant women are generally not advised to take oral preparations of Aloe vera because its cathartic property might result in stimulating uterine contractions, thereby increasing the risk for premature labor or miscarriage (Guo and Mei, 2016).

\section{CONCLUSION}

Sub-Saharan Africa is home to an extensive and diverse medicinal plant life. Consequently, the use of herbal medicines is widespread in that region. Despite this, the study of herbal medicine use during pregnancy which is related to maternal

\section{REFERENCES}

Abay, S. M., Lucantoni, L., Dahiya, N., Dori, G., Dembo, E. G., Esposito, F., et al. (2015). Plasmodium transmission blocking activities of Vernonia amygdalina extracts and isolated compounds. Malaria J. 14 (1), 288. doi: 10.1186/s12936015-0812-2

Abdullahi, A. A. (2011). Trends and challenges of traditional medicine in Africa. Afr. J. Tradit. Complement. Altern. Med. 8 (5 Suppl), 115-123. doi: 10.4314/ ajtcam.v8i5S.5

Adane, F., Seyoum, G., Alamneh, Y. M., Abie, W., Desta, M., and Sisay, B. (2020). Herbal medicine use and predictors among pregnant women attending antenatal care in Ethiopia: a systematic review and meta-analysis. $B M C$ Pregnancy Childbirth 20 (1), 157. doi: 10.1186/s12884-020-2856-8

Adegbehingbe, O. O., Adesanya, S. A., Idowu, T. O., Okimi, O. C., Oyelami, O. A., and Iwalewa, E. O. (2008). Clinical effects of Garcinia kola in knee osteoarthritis. J. Orthopaedic Surg. Res. 3, 34-34. doi: 10.1186/1749-799X-3-34

Adnan, M., Gul, S., Batool, S., Bibi, F., Rehman, A., Yaqoob, S., et al. (2017). A review on the ethnobotany, phytochemistry, pharmacology and nutritional composition of Cucurbita pepo L. J. Phytopharmacol. 6, 133-139.

Adusi-Poku, Y., Vanotoo, L., Detoh, E. K., Oduro, J., Nsiah, R. B., and Natogmah, A. Z. (2016). Type of herbal medicines utilized by pregnant women attending health, a public health priority in many sub-Saharan African countries, remains limited (Wekesah et al., 2016). Currently, there is not enough information to recommend the safe use of herbal medicinal products during pregnancy, as most herbal remedies used across sub-Saharan Africa are not backed by robust scientific studies. Moreover, several local herbal remedies used by sub-Saharan African pregnant women have never been botanically identified. This might be due to the lack of detailed documentation of traditional knowledge, which is generally transferred orally. Thus, further research on herbal medicine use during pregnancy is highly warranted in subSaharan Africa, as it might prevent maternal mortality and morbidity and decrease the possibility of endangering the health of fetuses.

A low disclosure rate of herbal medicine use to health care providers has also been identified in the present review. This suggests that collaborative communication by health care providers is important, as it may mitigate against the potential dangers of herbal medicine use during pregnancy and of uncoordinated concurrent herbal and conventional medicine use. Finally, given that traditional and sociocultural influence may play a role in herbal medicine use during pregnancy, there is a need to better understand the reasons and facilitators of herbal medicine use in pregnant women from sub-Saharan Africa.

\section{AUTHOR CONTRIBUTIONS}

$\mathrm{MH}$ drafted the manuscript. LH revised the manuscript.

\section{FUNDING}

This work was funded by the Centre for International Health, University of Bergen, Norway. ante-natal clinic in Offinso north district: Are orthodox prescribers aware? Ghana Med. J. 49, 227-232. doi: 10.4314/gmj.v49i4.2

Ahlberg, B. M. (2017). Integrated Health Care Systems and Indigenous Medicine: Reflections from the Sub-Sahara African Region. Front. Sociol. 2 (12), 1-10. doi: $10.3389 /$ fsoc. 2017.00012

Ahmed, M., Hwang, J. H., Hasan, M. A., and Han, D. (2018a). Herbal medicine use by pregnant women in Bangladesh: a cross-sectional study. BMC Complement. Altern. Med. 18 (1), 333. doi: 10.1186/s12906-018-2399-y

Ahmed, S. M., Nordeng, H., Sundby, J., Aragaw, Y. A., and de Boer, H. J. (2018b). The use of medicinal plants by pregnant women in Africa: A systematic review. J. Ethnopharmacol. 224, 297-313. doi: 10.1016/j.jep.2018.05.032

Al Disi, S. S., Anwar, M. A., and Eid, A. H. (2016). Anti-hypertensive Herbs and their Mechanisms of Action: Part I. Front. Pharmacol. 6, 323-323. doi: 10.3389/fphar.2015.00323

Alammar, N., Wang, L., Saberi, B., Nanavati, J., Holtmann, G., Shinohara, R. T. et al. (2019). The impact of peppermint oil on the irritable bowel syndrome: a meta-analysis of the pooled clinical data. BMC Complement. Altern. Med. 19 (1), 21. doi: 10.1186/s12906-018-2409-0

Allaire, A. D., Moos, M. K., and Wells, S. R. (2000). Complementary and alternative medicine in pregnancy: a survey of North Carolina certified nurse-midwives. Obstet. Gynecol. 95 (1), 19-23. doi: 10.1016/s0029-7844(99)00481-0 
Al-Ramahi, R., Jaradat, N., and Adawi, D. (2013). Use of herbal medicines during pregnancy in a group of Palestinian women. J. Ethnopharmacol. 150 (1), 79-84. doi: 10.1016/j.jep.2013.07.041

Al-Said, M. S., Tariq, M., Al-Yahya, M. A., Rafatullah, S., Ginnawi, O. T., and Ageel, A. M. (1990). Studies on Ruta chalepensis, an ancient medicinal herb still used in traditional medicine. J. Ethnopharmacol. 28 (3), 305-312. doi: 10.1016/0378-8741(90)90081-4

Alzohairy, M. A. (2016). Therapeutics Role of Azadirachta indica (Neem) and Their Active Constituents in Diseases Prevention and Treatment. EvidenceBased Complementary Altern. Med. : eCAM 2016, 7382506-7382506. doi: 10.1155/2016/7382506

Anwar, M. A., Al Disi, S. S., and Eid, A. H. (2016). Anti-Hypertensive Herbs and Their Mechanisms of Action: Part II. Front. Pharmacol. 7, 50-50. doi: 10.3389/ fphar.2016.00050

Atawodi, S. E., and Atawodi, J. C. (2009). Azadirachta indica (neem): a plant of multiple biological and pharmacological activities. Phytochem. Rev. 8 (3), 601620. doi: 10.1007/s11101-009-9144-6

Attah, A. F., O’Brien, M., Koehbach, J., Sonibare, M. A., Moody, J. O., Smith, T. J., et al. (2012). Uterine contractility of plants used to facilitate childbirth in Nigerian ethnomedicine. J. Ethnopharmacol. 143 (1), 377-382. doi: 10.1016/ j.jep.2012.06.042

Banda, Y., Chapman, V., Goldenberg, R. L., Stringer, J. S., Culhane, J. F., Sinkala, M., et al. (2007). Use of traditional medicine among pregnant women in Lusaka, Zambia. J. Altern. Complement. Med. 13 (1), 123-127. doi: 10.1089/ acm. 2006.6225

Bandyopadhyay, U., Biswas, K., Sengupta, A., Moitra, P., Dutta, P., Sarkar, D., et al. (2004). Clinical studies on the effect of Neem (Azadirachta indica) bark extract on gastric secretion and gastroduodenal ulcer. Life Sci. 75 (24), 2867-2878. doi: 10.1016/j.lfs.2004.04.050

Barnes, L. A. J., Barclay, L., McCaffery, K., and Aslani, P. (2018). Complementary medicine products used in pregnancy and lactation and an examination of the information sources accessed pertaining to maternal health literacy: a systematic review of qualitative studies. BMC Complement. Altern. Med. 18 (1), 229. doi: 10.1186/s12906-018-2283-9

Batisai, K. (2016). Towards an integrated approach to health and medicine in Africa. SAHARA J. : J. Soc. Aspects HIV/AIDS Res. Alliance 13 (1), 113-122. doi: $10.1080 / 17290376.2016 .1220323$

Bayisa, B., Tatiparthi, R., and Mulisa, E. (2014). Use of herbal medicine among pregnant women on antenatal care at nekemte hospital, Western ethiopia. Jundishapur J. Nat. Pharm. Prod. 9 (4), e17368. doi: 10.17795/jinpp-17368

Bent, S., Padula, A., Moore, D., Patterson, M., and Mehling, W. (2006). Valerian for sleep: a systematic review and meta-analysis. Am. J. Med. 119 (12), 10051012. doi: $10.1016 /$ j.amjmed.2006.02.026

Broussard, C. S., Louik, C., Honein, M. A., and Mitchell, A. A. (2010). Herbal use before and during pregnancy. Am. J. Obstet. Gynecol. 202 (5), 443.e441-446. doi: 10.1016/j.ajog.2009.10.865

Brower, V. (2008). Back to nature: extinction of medicinal plants threatens drug discovery. J. Natl. Cancer Inst. 100 (12), 838-839. doi: 10.1093/jnci/djn199

Challand, S., and Willcox, M. (2009). A clinical trial of the traditional medicine Vernonia amygdalina in the treatment of uncomplicated malaria. J. Altern. Complement. Med. 15 (11), 1231-1237. doi: 10.1089/acm.2009.0098

Cheang, K. I., Nguyen, T. T., Karjane, N. W., and Salley, K. E. (2016). Raspberry Leaf and Hypoglycemia in Gestational Diabetes Mellitus. Obstet. Gynecol. 128 (6), 1421-1424. doi: 10.1097/aog.0000000000001757

Chrubasik, S., Pittler, M. H., and Roufogalis, B. D. (2005). Zingiberis rhizoma: a comprehensive review on the ginger effect and efficacy profiles. Phytomedicine 12 (9), 684-701. doi: 10.1016/j.phymed.2004.07.009

Chuang, C. H., Chang, P. J., Hsieh, W. S., Tsai, Y. J., Lin, S. J., and Chen, P. C. (2009). Chinese herbal medicine use in Taiwan during pregnancy and the postpartum period: a population-based cohort study. Int. J. Nurs. Stud. 46 (6), 787-795. doi: 10.1016/j.ijnurstu.2008.12.015

Cuzzolin, L., Francini-Pesenti, F., Verlato, G., Joppi, M., Baldelli, P., and Benoni, G. (2010). Use of herbal products among 392 Italian pregnant women: focus on pregnancy outcome. Pharmacoepidemiol. Drug Saf. 19 (11), 1151-1158. doi: $10.1002 /$ pds. 2040

Dal'Belo, S. E., Gaspar, L. R., and Maia Campos, P. M. (2006). Moisturizing effect of cosmetic formulations containing Aloe vera extract in different concentrations assessed by skin bioengineering techniques. Skin Res. Technol. 12 (4), 241-246. doi: 10.1111/j.0909-752X.2006.00155.x

Dante, G., Bellei, G., Neri, I., and Facchinetti, F. (2014). Herbal therapies in pregnancy: what works? Curr. Opin. Obstet. Gynecol. 26 (2), 83-91. doi: $10.1097 / \mathrm{gco} .0000000000000052$

Dika, H., Dismas, M., Iddi, S., and Rumanyika, R. (2017). Prevalent use of herbs for reduction of labour duration in Mwanza, Tanzania: Are obstetricians aware? Tanzania J. Health Res. 19 (2), 1-8. doi: 10.4314/thrb.v19i2.5

Duru, C. B., Uwakwe, K. A., Chinomnso, N. C., Mbachi, I. I., Diwe, K. C., Agunwa, C. C., et al. (2016). Socio-demographic Determinants of Herbal Medicine Use in Pregnancy Among Nigerian Women Attending Clinics in a Tertiary Hospital in Imo State, South-East, Nigeria. Am. J. Med. Stud. 4 (1), 1-10. doi: 10.12691/ajms-4-1-1

Ernst, E. (2002). Herbal medicinal products during pregnancy: are they safe? Bjog 109 (3), 227-235. doi: 10.1111/j.1471-0528.2002.t01-1-01009.x

Facchinetti, F., Pedrielli, G., Benoni, G., Joppi, M., Verlato, G., Dante, G., et al. (2012). Herbal supplements in pregnancy: unexpected results from a multicentre study. Hum. Reprod. 27 (11), 3161-3167. doi: 10.1093/humrep/ $\operatorname{des} 303$

Fakeye, T. O., Adisa, R., and Musa, I. E. (2009). Attitude and use of herbal medicines among pregnant women in Nigeria. BMC Complementary Altern. Med. 9, 53-53. doi: 10.1186/1472-6882-9-53

Fruscalzo, A., Salmeri, M. G., Cendron, A., Londero, A. P., and Zanni, G. (2012). Introducing routine trial of labour after caesarean section in a second level hospital setting. J. Mater. Fetal Neonatal Med. 25 (8), 1442-1446. doi: 10.3109/ 14767058.2011.640367

Gakuya, D. W., Okumu, M. O., Kiama, S. G., Mbaria, J. M., Gathumbi, P. K., Mathiu, P. M., et al. (2020). Traditional medicine in kenya: past and current status, challenges and the way forward. Sci. Afr. 8, e00360. doi: 10.1016/ j.sciaf.2020.e00360

Gebremedhin, G., and Gomathi, P. (2014). Assessment of drug use and effect in pregnant women attending antenatal care in hospitals of Mekelle, Tigray, Ethiopia. J. Drug Deliv. Ther. 4 (6), 75-82. doi: 10.22270/jddt.v4i6.999

Gilad, R., Hochner, H., Savitsky, B., Porat, S., and Hochner-Celnikier, D. (2018). Castor oil for induction of labor in post-date pregnancies: A randomized controlled trial. Women Birth 31 (1), e26-e31. doi: 10.1016/ j.wombi.2017.06.010

Godlove, M. (2011). Prevalence of herbal medicine use and associated factors among pregnant women attending antenatal clinic at Mbeya Refferal Hospital in 2010 (Dar es Salaam, TZ: Muhimbili University of Health and Allied Sciences).

Govaerts, R. (2001). How many species of seed plants are there? TAXON 50 (4), 1085-1090. doi: 10.2307/1224723

Govindachari, T. R., Suresh, G., Gopalakrishnan, G., Banumathy, B., and Masilamani, S. (1998). Identification of antifungal compounds from the seed oil ofAzadirachta Indica. Phytoparasitica 26 (2), 109-116. doi: 10.1007/ BF02980677

Guo, X., and Mei, N. (2016). Aloe vera: A review of toxicity and adverse clinical effects. J. Environ. Sci. Health C Environ. Carcinog. Ecotoxicol. Rev. 34 (2), $77-$ 96. doi: 10.1080/10590501.2016.1166826

Hailemeskel, B., Fullas, F., Habte, A., Al-Matari, R., and Brewer, D. (2017). A review of natural remedies commonly used by Ethiopian immigrants in the USA. Curr. Res.: Integr. Med. 2, 31-36. doi: 10.4172/2529-797X.1000024

Heitmann, K., Nordeng, H., and Holst, L. (2013). Pregnancy outcome after use of cranberry in pregnancy-the Norwegian Mother and Child Cohort Study. BMC Complement. Altern. Med. 13, 345. doi: 10.1186/1472-6882-13-345

Hekmatpou, D., Mehrabi, F., Rahzani, K., and Aminiyan, A. (2019). The Effect of Aloe Vera Clinical Trials on Prevention and Healing of Skin Wound: A Systematic Review. Iranian J. Med. Sci. 44 (1), 1-9.

Holst, L., Wright, D., Haavik, S., and Nordeng, H. (2011). Safety and efficacy of herbal remedies in obstetrics-review and clinical implications. Midwifery 27 (1), 80-86. doi: 10.1016/j.midw.2009.05.010

Hwang, J. H., Kim, Y.-R., Ahmed, M., Choi, S., Al-Hammadi, N. Q., Widad, N. M., et al. (2016). Use of complementary and alternative medicine in pregnancy: a cross-sectional survey on Iraqi women. BMC Complementary Altern. Med. 16, 191-191. doi: 10.1186/s12906-016-1167-0

Illamola, S. M., Amaeze, O. U., Krepkova, L. V., Birnbaum, A. K., Karanam, A., Job, K. M., et al. (2020). Use of Herbal Medicine by Pregnant Women: What 
Physicians Need to Know. Front. Pharmacol. 10, 1483-1483. doi: 10.3389/ fphar.2019.01483

International Bioethics Committee (2013). Report of the IBC on traditional medicine systems and their ethical implications, [Online]. Paris: UNESCO. Available: https://unesdoc.unesco.org/ark:/48223/pf0000217457 [Accessed February 23, 2020].

Isa, A., Gaya, B., Saleh, M., Mohammed, A., Muhammad, A., A, M., et al. (2014). The Modulatory Role of Aqueous Extract of Vernonia Amygdalina Extract on Pregnant Rats. IOSR J. Pharm. Biol. Sci. 9 (3), 45-47. doi: 10.9790/300809324547

Jafarian, A., Zolfaghari, B., and Parnianifard, M. (2012). The effects of methanolic, chloroform, and ethylacetate extracts of the Cucurbita pepo L. on the delay type hypersensitivity and antibody production. Res. Pharmaceut. Sci. 7 (4), $217-224$.

James, P. B., Bah, A. J., Tommy, M. S., Wardle, J., and Steel, A. (2018a). Herbal medicines use during pregnancy in Sierra Leone: An exploratory crosssectional study. Women Birth 31 (5), e302-e309. doi: 10.1016/ j.wombi.2017.12.006

James, P. B., Wardle, J., Steel, A., and Adams, J. (2018b). Traditional, complementary and alternative medicine use in Sub-Saharan Africa: a systematic review. BMJ Glob. Health 3 (5), e000895. doi: 10.1136/bmjgh2018-000895

John, L. J., and Shantakumari, N. (2015). Herbal Medicines Use During Pregnancy: A Review from the Middle East. Oman Med. J. 30 (4), 229-236. doi: $10.5001 /$ omj. 2015.48

Kaingu, C. K., Oduma, J. A., and Kanui, T. I. (2011). Practices of traditional birth attendants in Machakos District, kenya. J. Ethnopharmacol. 137 (1), 495-502. doi: 10.1016/j.jep.2011.05.044

Kefe, A., Giday, M., Mamo, H., and Erko, B. (2016). Antimalarial properties of crude extracts of seeds of Brucea antidysenterica and leaves of Ocimum lamiifolium. BMC Complement. Altern. Med. 16 (1), 118. doi: 10.1186/ s12906-016-1098-9

Kennedy, D. A., Lupattelli, A., Koren, G., and Nordeng, H. (2013). Herbal medicine use in pregnancy: results of a multinational study. $B M C$ Complement. Altern. Med. 13, 355. doi: 10.1186/1472-6882-13-355

Kennedy, D. A., Lupattelli, A., Koren, G., and Nordeng, H. (2016). Safety classification of herbal medicines used in pregnancy in a multinational study. BMC Complement. Altern. Med. 16, 102. doi: 10.1186/s12906-0161079-z

Khadivzadeh, T., and Ghabel, M. (2012). Complementary and alternative medicine use in pregnancy in Mashhad, Iran 2007-8. Iran J. Nurs. Midwifery Res. 17 (4), 263-269.

Kumar, V. S., and Navaratnam, V. (2013). Neem (Azadirachta indica): prehistory to contemporary medicinal uses to humankind. Asian Pacific J. Trop. Biomed. 3 (7), 505-514. doi: 10.1016/S2221-1691(13)60105-7

Laelago, T., Yohannes, T., and Lemango, F. (2016). Prevalence of herbal medicine use and associated factors among pregnant women attending antenatal care at public health facilities in Hossana Town, Southern Ethiopia: facility based cross sectional study. Arch. Public Health 74, 7. doi: 10.1186/s13690-016-0118-z

Laelago, T. (2018). "Herbal Medicine Use during Pregnancy: Benefits and Untoward Effects," in Herbal Medicine. Ed. P. Builders. London: IntechOpen.

Luo, X., Jiang, Y., Fronczek, F. R., Lin, C., Izevbigie, E. B., and Lee, K. S. (2011). Isolation and structure determination of a sesquiterpene lactone (vernodalinol) from Vernonia amygdalina extracts. Pharm. Biol. 49 (5), 464-470. doi: $10.3109 / 13880209.2010 .523429$

Luong, K., Dunkel, F. V., Coulibaly, K., and Beckage, N. E. (2012). Potential use of neem leaf slurry as a sustainable dry season management strategy to control the malaria vector Anopheles gambiae (DIPTERA: CULICIDAE) in west African villages. J. Med. Entomol. 49 (6), 1361-1369. doi: 10.1603/me12075

Lusaka Times (2018). ZAMRA to stiffen regulation of herbal medicine, [Online]. Lusaka, ZM: Lusaka Times. Available: https://www.lusakatimes.com/2018/06/ 19/zamra-to-stiffen-regulation-of-herbal-medicine/ [Accessed February 23, 2020].

Mahmoodpoor, A., Medghalchi, M., Nazemiyeh, H., Asgharian, P., Shadvar, K., and Hamishehkar, H. (2018). Effect of Cucurbita Maxima on Control of Blood Glucose in Diabetic Critically Ill Patients. Adv. Pharmaceut. Bull. 8 (2), 347351. doi: 10.15171/apb.2018.040
Malan, D. F., Neuba, D. F. R., and Kouakou, K. L. (2015). Medicinal plants and traditional healing practices in ehotile people, around the aby lagoon (eastern littoral of Côte d'Ivoire). J. Ethnobiol. Ethnomed. 11 (1), 21. doi: 10.1186/ s13002-015-0004-8

Maluma, S., Kalungia, A. C., Hamachila, A., Hangoma, J., and Munkombwe, D. (2017). Prevalence of Traditional Herbal Medicine use and associated factors among pregnant women of Lusaka Province, Zambia. J. Preventive Rehabilitative Med. 1, 5-11. doi: 10.21617/jprm.2017.0102.1

Mawoza, T., Nhachi, C., and Magwali, T. (2019). Prevalence of Traditional Medicine Use during Pregnancy, at Labour and for Postpartum Care in a Rural Area in Zimbabwe. Clinics Mother Child Health 16 (2), 321. doi: 10.24105/2090-7214.16.321

Mekuria, A. B., Erku, D. A., Gebresillassie, B. M., Birru, E. M., Tizazu, B., and Ahmedin, A. (2017). Prevalence and associated factors of herbal medicine use among pregnant women on antenatal care follow-up at University of Gondar referral and teaching hospital, Ethiopia: a cross-sectional study. BMC Complement. Altern. Med. 17 (1), 86. doi: 10.1186/s12906-017-1608-4

Mensah, M., Komlaga, G., Donkor, A., Firempong, C., Anning, A., and Dickson, R. (2019). "Toxicity and Safety Implications of Herbal Medicines Used in Africa," in Herbal Medicine. Ed. P. Builders. London: IntechOpen.

Mishra, A., and Dave, N. (2013). Neem oil poisoning: Case report of an adult with toxic encephalopathy. Indian J. Crit. Care Med. 17 (5), 321-322. doi: 10.4103/ 0972-5229.120330

Moshi, M., and Mhame, P. (2013). "Legislation on Medicinal Plants in Africa," in Medicinal Plant Research in Africa: Pharmacology and Chemistry. Ed. V. Kuete. Amsterdam: Elsevier.

Mothupi, M. C. (2014). Use of herbal medicine during pregnancy among women with access to public healthcare in Nairobi, Kenya: a cross-sectional survey. BMC Complement. Altern. Med. 14, 432. doi: 10.1186/1472-6882-14-432

Munoz Balbontin, Y., Stewart, D., Shetty, A., Fitton, C. A., and McLay, J. S. (2019). Herbal Medicinal Product Use During Pregnancy and the Postnatal Period: A Systematic Review. Obstet. Gynecol. 133 (5), 920-932. doi: 10.1097/ aog.0000000000003217

Mureyi, D. D., Monera, T. G., and Maponga, C. C. (2012). Prevalence and patterns of prenatal use of traditional medicine among women at selected harare clinics: a cross-sectional study. BMC Complement. Altern. Med. 12, 164. doi: 10.1186/ $1472-6882-12-164$

Myhre, R., Brantsaeter, A. L., Myking, S., Eggesbo, M., Meltzer, H. M., Haugen, M., et al. (2013). Intakes of garlic and dried fruits are associated with lower risk of spontaneous preterm delivery. J. Nutr. 143 (7), 1100-1108. doi: 10.3945/ jn.112.173229

Nalumansi, P. A., Kamatenesi-Mugisha, M., and Anywar, G. (2017). Medicinal Plants used during Antenatal Care by Pregnant Women in Eastern Uganda. Afr. J. Reprod. Health 21 (4), 33-44. doi: 10.29063/ajrh2017/v21i4.4

Nega, S. S., Bekele, H. M., Meles, G. G., and Nordeng, H. (2019). Medicinal Plants and Concomitant Use with Pharmaceutical Drugs Among Pregnant Women. J. Altern. Complement. Med. 25 (4), 427-434. doi: 10.1089/acm.2018.0062

Nergard, C. S., Ho, T. P., Diallo, D., Ballo, N., Paulsen, B. S., and Nordeng, H. (2015). Attitudes and use of medicinal plants during pregnancy among women at health care centers in three regions of Mali, West-Africa. J. Ethnobiol. Ethnomed. 11, 73. doi: 10.1186/s13002-015-0057-8

Nordeng, H., and Havnen, G. C. (2004). Use of herbal drugs in pregnancy: a survey among 400 Norwegian women. Pharmacoepidemiol. Drug Saf. 13 (6), 371-380. doi: $10.1002 /$ pds. 945

Nordeng, H., Bayne, K., Havnen, G. C., and Paulsen, B. S. (2011). Use of herbal drugs during pregnancy among 600 Norwegian women in relation to concurrent use of conventional drugs and pregnancy outcome. Complement. Ther. Clin. Pract. 17 (3), 147-151. doi: 10.1016/j.ctcp.2010.09.002

Nyeko, R., Tumwesigye, N. M., and Halage, A. A. (2016). Prevalence and factors associated with use of herbal medicines during pregnancy among women attending postnatal clinics in Gulu district, Northern Uganda. BMC Pregnancy Childbirth 16 (1), 296-296. doi: 10.1186/s12884-016-1095-5

Okoye, T. C., Uzor, P. F., Onyeto, C. A., and Okereke, E. K. (2014). "18 - Safe African Medicinal Plants for Clinical Studies," in Toxicological Survey of African Medicinal Plants. Ed. V. Kuete (Amsterdam: Elsevier), 535-555.

Okumu, M. O., Ochola, F. O., Onyango, A. O., Mbaria, J. M., Gakuya, D. W., Kanja, L. W., et al. (2017). The legislative and regulatory framework governing 
herbal medicine use and practice in Kenya: a review. Pan. Afr. Med. J. 28, 232. doi: 10.11604/pamj.2017.28.232.12585

Olawoye, O. S., Adeagbo, B. A., and Bolaji, O. O. (2018). Moringa oleifera leaf powder alters the pharmacokinetics of amodiaquine in healthy human volunteers. J. Clin. Pharm. Ther. 43 (5), 626-632. doi: 10.1111/jcpt.12725

Olowokere, A., and Olajide, O. (2013). Women's perception of safety and utilization of herbal remedies during pregnancy in a local government area in Nigeria. Clin. Nurs. Stud. 1, 9-22. doi: 10.5430/cns.vln4p9

Orief, Y. I., Farghaly, N. F., and Ibrahim, M. I. A. (2014). Use of herbal medicines among pregnant women attending family health centers in Alexandria. Middle East Fertility Soc. J. 19 (1), 42-50. doi: 10.1016/j.mefs.2012.02.007

Oyeyemi, I. T., Akinlabi, A. A., Adewumi, A., Aleshinloye, A. O., and Oyeyemi, O. T. (2018). Vernonia amygdalina: A folkloric herb with anthelminthic properties. BeniSuef Univ. J. Basic Appl. Sci. 7 (1), 43-49. doi: 10.1016/j.bjbas.2017.07.007

Ozioma, E., and Chinwe, O. (2019). "Herbal Medicines in African Traditional Medicine," in Herbal Medicine. Ed. P. Builders. London: IntechOpen.

Pai, M. R., Acharya, L. D., and Udupa, N. (2004). Evaluation of antiplaque activity of Azadirachta indica leaf extract gel-a 6-week clinical study. J. Ethnopharmacol. 90 (1), 99-103. doi: 10.1016/j.jep.2003.09.035

Peprah, P., Agyemang-Duah, W., Arthur-Holmes, F., Budu, H. I., Abalo, E. M., Okwei, R., et al. (2019). 'We are nothing without herbs': a story of herbal remedies use during pregnancy in rural Ghana. BMC Complement. Altern. Med. 19 (1), 65. doi: 10.1186/s12906-019-2476-x

Pretorius, E. (1991). Traditional and modern medicine working in tandem. Curationis 14 (4), 10-13. doi: 10.4102/curationis.v14i4.339

Sahalie, N. A., Abrha, L. H., and Tolesa, L. D. (2018). Chemical composition and antimicrobial activity of leave extract of Ocimum lamiifolium (Damakese) as a treatment for urinary tract infection. Cogent Chem. 4 (1), 1440894. doi: $10.1080 / 23312009.2018 .1440894$

Saper, R. B., Kales, S. N., Paquin, J., Burns, M. J., Eisenberg, D. M., Davis, R. B., et al. (2004). Heavy metal content of ayurvedic herbal medicine products. JAMA 292 (23), 2868-2873. doi: 10.1001/jama.292.23.2868

Shewamene, Z., Dune, T., and Smith, C. A. (2017). The use of traditional medicine in maternity care among African women in Africa and the diaspora: a systematic review. BMC Complement. Altern. Med. 17 (1), 382. doi: 10.1186/ s12906-017-1886-x

Sicuranza, G. B., and Figueroa, R. (2003). Uterine rupture associated with castor oil ingestion. J. Mater. Fetal Neonatal Med. 13 (2), 133-134. doi: 10.1080/jmf.13.2.133.134

Silva, F. V., Dias, F., Costa, G., and Campos, M. D. G. (2018). Chamomile reveals to be a potent galactogogue: the unexpected effect. J. Mater. Fetal Neonatal Med. 31 (1), 116-118. doi: 10.1080/14767058.2016.1274300

Sinniah, D., and Baskaran, G. (1981). Margosa oil poisoning as a cause of Reye's syndrome. Lancet 1 (8218), 487-489. doi: 10.1016/s0140-6736(81)91861-4

Stephani, V., Opoku, D., and Beran, D. (2018). Self-management of diabetes in Sub-Saharan Africa: a systematic review. BMC Public Health 18 (1), 1148. doi: 10.1186/s12889-018-6050-0

Sundaravalli, N., Raju, B. B., and Krishnamoorthy, K. A. (1982). Neem oil poisoning. Indian J. Pediatr. 49 (398), 357-359. doi: 10.1007/bf02834422

Talwar, G. P., Shah, S., Mukherjee, S., and Chabra, R. (1997). Induced termination of pregnancy by purified extracts of Azadirachta Indica (Neem): mechanisms involved. Am. J. Reprod. Immunol. 37 (6), 485-491. doi: 10.1111/j.16000897.1997.tb00264.x

Tamuno, I., Omole-Ohonsi, A., and Fadare, J. (2010). Use Of Herbal Medicine Among Pregnant Women Attending A Tertiary Hospital In Northern Nigeria. Internet J. Gynecol. Obstetr. 15 (2), 1-8. doi: 10.5580/2932
Trabace, L., Tucci, P., Ciuffreda, L., Matteo, M., Fortunato, F., Campolongo, P., et al. (2015). "Natural" relief of pregnancy-related symptoms and neonatal outcomes: above all do no harm. J. Ethnopharmacol. 174, 396-402. doi: 10.1016/j.jep.2015.08.046

Tunaru, S., Althoff, T. F., Nüsing, R. M., Diener, M., and Offermanns, S. (2012). Castor oil induces laxation and uterus contraction via ricinoleic acid activating prostaglandin EP3 receptors. Proc. Natl. Acad. Sci. U. States America 109 (23), 9179-9184. doi: 10.1073/pnas.1201627109

Viljoen, E., Visser, J., Koen, N., and Musekiwa, A. (2014). A systematic review and meta-analysis of the effect and safety of ginger in the treatment of pregnancyassociated nausea and vomiting. Nutr. J. 13, 20. doi: 10.1186/1475-2891-13-20

Wachtel-Galor, S., and Benzie, I. F. F. (2011). "Herbal Medicine: An Introduction to Its History, Usage, Regulation, Current Trends, and Research Needs," in Herbal Medicine: Biomolecular and Clinical Aspects. Eds. I. F. F. Benzie and S. Wachtel-Galor (Boca Raton FL: CRC Press/Taylor \& Francis Llc).

Wang, H. P., Yang, J., Qin, L. Q., and Yang, X. J. (2015). Effect of garlic on blood pressure: a meta-analysis. J. Clin. Hypertens. (Greenwich) 17 (3), 223-231. doi: $10.1111 /$ jch. 12473

Wekesah, F. M., Mbada, C. E., Muula, A. S., Kabiru, C. W., Muthuri, S. K., and Izugbara, C. O. (2016). Effective non-drug interventions for improving outcomes and quality of maternal health care in sub-Saharan Africa: a systematic review. Syst. Rev. 5 (1), 137. doi: 10.1186/s13643-016-0305-6

World Health Organization (2005). National Policy on Traditional Medicine and Regulation of Herbal Medicines - Report of a WHO Global Survey, [Online]. Geneva: World Health Organization. Available: https://apps.who.int/iris/ bitstream/handle/10665/43229/9241593237.pdf?sequence $=1$ [Accessed February 23, 2020].

Xia, H. C., Li, F., Li, Z., and Zhang, Z. C. (2003). Purification and characterization of Moschatin, a novel type I ribosome-inactivating protein from the mature seeds of pumpkin (Cucurbita moschata), and preparation of its immunotoxin against human melanoma cells. Cell Res. 13 (5), 369-374. doi: 10.1038/sj.cr.7290182

Zamawe, C., King, C., Jennings, H. M., Mandiwa, C., and Fottrell, E. (2018). Effectiveness and safety of herbal medicines for induction of labour: a systematic review and meta-analysis. BMJ Open 8 (10), e022499-e022499. doi: 10.1136/bmjopen-2018-022499

Zeichen de Sa, R., Rey, A., Arganaraz, E., and Bindstein, E. (2000). Perinatal toxicology of Ruta chalepensis (Rutaceae) in mice. J. Ethnopharmacol. 69 (2), 93-98. doi: 10.1016/s0378-8741(98)00232-3

Ziaei, S., Hantoshzadeh, S., Rezasoltani, P., and Lamyian, M. (2001). The effect of garlic tablet on plasma lipids and platelet aggregation in nulliparous pregnants at high risk of preeclampsia. Eur. J. Obstet. Gynecol. Reprod. Biol. 99 (2), 201206. doi: 10.1016/s0301-2115(01)00384-0

\section{Conflict of Interest: MH is a Partner 4 Health employee.}

The remaining author declares that the research was conducted in the absence of any commercial or financial relationships that could be construed as a potential conflict of interest.

Copyright $\odot 2020 \mathrm{El} \mathrm{Hajj}$ and Holst. This is an open-access article distributed under the terms of the Creative Commons Attribution License (CC BY). The use, distribution or reproduction in other forums is permitted, provided the original author $(s)$ and the copyright owner(s) are credited and that the original publication in this journal is cited, in accordance with accepted academic practice. No use, distribution or reproduction is permitted which does not comply with these terms. 